\title{
Infectious Dynamics in Urban 3D-Environment: Challenges and Possibilities*
}

\author{
Eugene Eremchenko [0000-0002-4416-7617], Vladimir Tikunov ${ }^{10000-0002-1597-6909]}$, \\ Sergey Vylegzhanin ${ }^{20000-0002-4259-9811]}$ and Alexander Fetishev ${ }^{3[0000-0003-3420-6552]}$ \\ ${ }^{1}$ Lomonosov Moscow State University, Moscow GSP-1, 119991, Russia, \\ ${ }^{2}$ MRUNo174FMBA, Protvino, Russia, \\ ${ }^{3}$ Rospotrebnadzor Center No174, Protvino, Russia \\ eugene.eremchenko@gmail.com
}

\begin{abstract}
The article is a brief overview of the results of the current stage of study of the dynamics of infectious processes in the modern urban environment in Protvino (Russia) with the help of 3D-model of the town, made in the paradigm of the Digital Earth. Data for the period 2011-2016 (38791 events) were used. Spatial and temporal resolution "building-day" was achieved. It is demonstrated that infectious diseases rates even in neighboring buildings can vary significantly from one to another. The presence of buildings with both significantly higher and significantly lower rates of infectious diseases is shown. Such significant discrepancies between rates are difficult to explain by local ecological factors like air and water pollutions because of the generalized nature of their impact on such a small area. The example of the global dynamics of COVID-19 demonstrates the need to study both specific and non-specific factors for the local epidemic process. Prospectives of the future researches are discussed briefly.
\end{abstract}

Keywords: 3D-model, Urban Environment, Infection Diseases, Digital Earth, COVID-19

\section{Introduction}

The comprehensive study of the development of infectious processes in modern urban environment is highly urgent, which is clearly demonstrated by the pandemic COVID19 [1]. In addition to practical relevance, this area of work also has an undoubted fundamental importance, as it provides an opportunity to obtain new knowledge about the

Copyright (C) 2020 for this paper by its authors. Use permitted under Creative Commons License Attribution 4.0 International (CC BY 4.0).

* Supported by RFBR grant \#18-05-00236 (preprocessing of data) and RSF grant \#20-47-01001 (data analysis, investigations, discussion and conclusions) 


\section{E. Eremchenko, V. Tikunov, S. Vylegzhanin, et al.}

nature of the infectious diseases and other social processes based on analysis of empirical material. At the same time, the solution of this problem is hindered by serious methodological challenges.

High population density and its volatility in a modern city essentially impacts the distribution and the dynamics of infections, and considerable change of living conditions in combination with deterioration of the environment and deep changes of the ecology, high psycho-emotional loadings, deformation of habitual rhythm of life and other factors complicates this problem even more. In the absence of satisfactory theoretical models describing social processes in general and the development of epidemics in particular, understanding the situation by the means of empirical data plays a special role. However, numerous problems also arise.

In general, we could assume that the recording of infectious diseases in a modern city is organized with sufficient efficiency and accuracy. At the same time, perception of the spatial and temporal aspect of these data is complicated by the lack of a context in which it can be aggregated, stored and processed.

As a rule, the information about diseases is represented mainly in the form of temporally organized lists. Extracting spatial data from it is a rather complicated procedure due to lack of the sufficient geospatial context. In addition, residential areas of modern cities include multi-stores buildings, and the representation of data on the development of infectious processes in them requires the availability of high-quality and usable three-dimensional models of that buildings, which are very rare at present. We can say that the problem of studying epidemic processes in the modern urban environment requires the seamlessly integrated three-dimensional information environments that allow visual perception with very high spatial and temporal resolutions and within rich and updated geospatial context.

This problem is quite obvious and has been understood for a long time, and the spatial and temporal approach is widely used in the study of infectious processes [2]. As early as the 19th century, John Snow convincingly demonstrated the effectiveness of spatial analysis of infectious processes using a geoinformation system based on a largescale map when studying the cholera epidemic in London in 1854. [3]. However, this kind of analysis is usually provided ad hoc in emergency situations of the outbreaks of dangerous infections. Systematic analysis of infectious and social processes in general with high spatial and temporal resolution is still a complex and largely unsolved task. This, in turn, generates significant methodological difficulties in the study of infectious processes leading to deep systematic errors.

1. There is no regular detailed spatio-temporal studies of the processes of infection development in the urban environment in all its diversity. The extreme complexities of such studies leads to the fact that there is a threshold when modern methods of spatial research begin to be involved, that leads to biased vision of the process.

2. Lack of basis for such modeling - high-precision models of the urban environment, allowing analysis with a reasonable and technically achievable level of spatial and temporal resolution. This leads to significant data generalization and, as a result, to systematic data distortions. 
3. An important factor of possible systematic errors in perception of the situation is the inability to integrate spatial data in the mapping environment without deterioration of data. Even in the presence of highly accurate data for cities and territories their integration by cartographic means is impossible without generalization with irreversible loss of initial accuracy of raw data. This makes impossible studying phenomena beyond the classical model of the social processes - for example, fractal relationships, correlations in space and time and synchronous effects, etc. In fact, the cartographic approach fits well to current model of infection that developed through locally acting material agents - viruses or bacteria - but complicates the comparative analysis of alternative models.

4. Fourth, combining raw data with cartographic context makes it difficult to study the dependence of the medical and environmental situations on factors like terrain. Generalization of the data about the medical and social environment is methodically questionable and excludes the possibility of studying processes characterized by high variability of space-time parameters.

5. Fifth, the impossibility or complexity of seamless integration of spatial data without loss of accuracy complicates the formation of generalizing sets of empirical materials necessary to study a complex social phenomenon.

In fact, the resolution of registration of infectious diseases already achieved in urban conditions is "apartment - day". However, this information is accumulated in the form of lists; its projection into a geospatial context using a cartographic approach is significantly complicated, time-consuming, and makes the seamless integration of data from different cities and territories impossible. It is the visualization and spatial and temporal analysis of already available data that becomes a "narrow neck" on the way to systematic study of already accumulated empirical material. Resolution of these issues requires a critical analysis of existing methodological tools and approaches to the visualization of infectious, as well as social in general, processes with the highest possible spatial and temporal resolution.

\section{Methodology}

\subsection{Methodology of data aggregation and visualization}

Qualitative transformation of geospatial methods at the turn of the XXI century raised the question of the need to create a typology of methods of geospatial imaging and allowed to distinguish four main types: 1) maps (and cartographic GIS), 2) atlases and geoportals of Google.Maps class, 3) globes, 4) Digital Earth. The ability to support different viewing angles of the object and its distances can be used as a classification criterion. They, in turn, may be shown with the help of diagrams "angle - range" [4]. Only Digital Earth as a special method of geospatial visualization supports visualization from any possible angle ( $4 \pi$ geometry) and from any possible distance.

Even in Google Earth - first online service, in which Digital Earth paradigm was implemented (Google Earth, 2005 [5]), this functionality was implemented in full, and the choice of scale was limited only by the spatial resolution of available at the time of 
remote sensing data and could be estimated in eight orders of magnitude, which obviously overlapped the whole range of actual cartographic scales. The requirement of multiscale representation, which was laid down in the Digital Earth concept initially [6], was overcome - instead of a discrete set of several scales whole ranges of scales and sights of view were supported, which radically expanded heuristic possibilities of the system [7]. Accurate and global analysis unconfined to scale and projection could be possible only within the Digital Earth paradigm.

\subsection{Methodology of analysis}

In the situation of incompleteness of existing models of biological and social processes, the role of visual analytics as a means of primary analysis of raw data preceding the choice of a theoretical model is especially significant. Therefore, it is important to choose the dominant methodological approach for describing the dynamics of infectious processes. As such, it makes sense to choose a combination of searching for both specific and non-specific features of their spatial and temporal dynamics.

It is natural for the analysis of processes in an urban environment to search for specific features to a given spatial extent and time horizon. However, the urban environment is not a closed system - the city interacts with the planet as a whole through intensive economic activity and exchange of goods, regular migration, information exchange, tourism, etc. The environmental factor that affects the processes in the city, also can not be considered as deliberately local. The development of transport and tourism makes possible the rapid spread of viruses and bacteria throughout the planet.

Therefore, it seems reasonable and desirable to analyze information about infectious processes in the urban environment not to consider the city as a system deliberately isolated from the rest of the world and, therefore, to take into account the non-specific features of the processes under study.

The effectiveness of the methodology for studying non-specific features of processes was clearly demonstrated when Hans Selje discovered and studied adaptation syndrome (stress) [8]. This fruitful approach can also be applied to the study of spatial and temporal dynamics of infectious processes and environment.

\section{Research}

As the object of study was chosen Protvino. It is a relatively small and young (60 years old) town with the population of about 35 thousand people and an area of about 27 square kilometers, located in the forest area of Serpukhovsky district of Moscow region, $100 \mathrm{~km}$ south of Moscow, on the bank of Protva river. Protvino is a well-known scientific center of high energy physics.

The choice was dictated by a number of circumstances:

- The compactness and the stability of the town. The relative remoteness from Moscow, the absence of a railway station in the town reduce the impact of migration, which complicate the long-term analysis of social processes. 
- High quality and homogenity of the town infrastructure. Protvino is a relatively young town with residential buildings from a few projects. There are no spatially isolated and the deprived areas in the town.

- The environmental situation in the town is relatively favorable. There are no hazardous industries in the town. Protvino is located in the forest, air is clean. Roads are relatively good. Water and energy supplies are in good conditions. Removal of domestic wastes is well organized. 3D-model of Protvino was made in 2007

\subsection{Protvino 3D-model}

Three-dimensional model of Protvino was created in the paradigm of the Digital Earth. It succeeds to map and model of the town, created since 2001 by the means of GIS on the base of erected polygons. With the appearance of Google Earth service in 2005, the town-wide model was created in the KML format and became the first open city model in Russia in 2007 (Fig. 1).

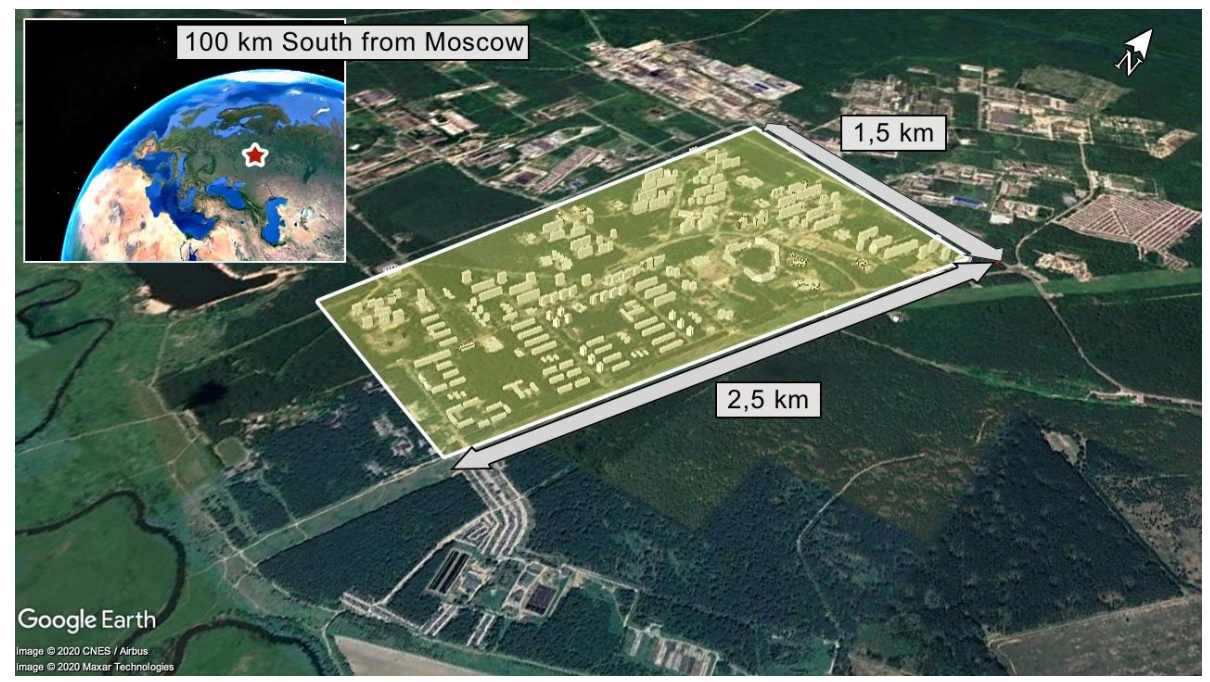

Fig. 1. 3D-model of the residential area of the Protvino, Russia, immersed in the Google Earth geospatial context.

From 2008 until the termination of Google Earth API in 2017, the model was also available online at Vprotvino.ru [9]. The Protvino model was developed in the following directions:

1. expansion of the extent (inclusion of new territories);

2. update of information on buildings and structures;

3. model refinement using remote sensing data;

4. creating the heterogeneous dataset consists of different information (road network, business infrastructure, points of interests, etc.);

5. providing the photovisual models of the buildings; 
6 E. Eremchenko, V. Tikunov, S. Vylegzhanin, et al.

6. providing spheric panoramas (like Google's Street View);

7. using model for the case studies.
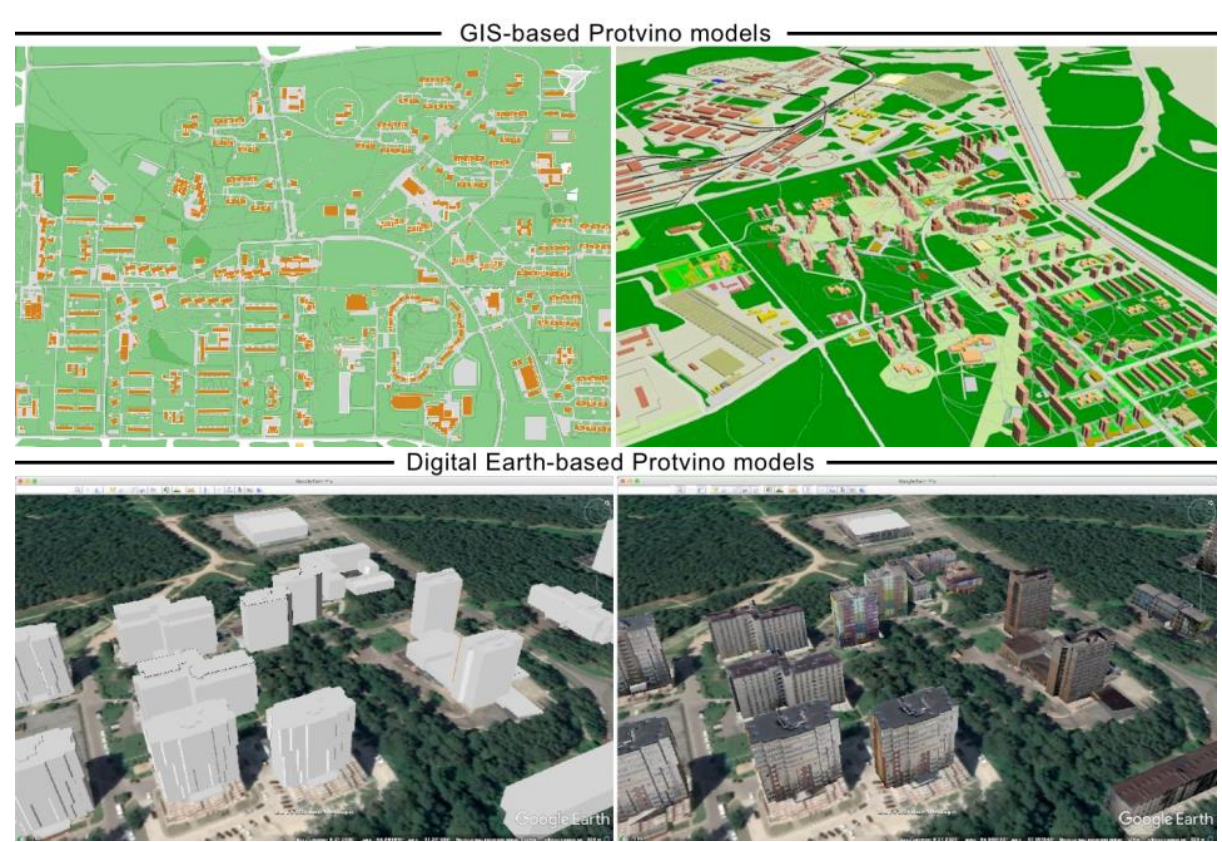

Fig. 2. (a - d). (Left to right, up to down,). Different stages of developing of Protvino model: a) 2D- model Protvino (2001-2004); b) 3D GIS of Protvino (2004-2005); c) Simple Digital Earth Protvino model (2005-2008); d) Digital Earth Protvino model with rich photovisualization of the buildings (2014-2020).

To create 3D models SketchUp was used, as well as open data - photos of buildings and structures taken by ordinary household cameras, and geospatial context provided by Google Earth. With this approach, it is possible to provide high performance and relative accuracy of restoration of the geometry of the models up to a few percents, as well as assures their excellent visual recognition and high accuracy of their positioning in an urban terrain (about few meters). In the first stage of the work, photographs were used to restore the geometry of buildings and their localization, and later photographs were used for photovisualization as well.

At present (2020) the model covers the entire main residential part of the town near $2.5 \times 1.5 \mathrm{~km}$ and is periodically updated as new buildings and structures appear. Photovisualization of the models provides the context for accurate localization of social events (especially those registered outside the buildings and structures), as well as effective perception of visual information. At present, about $40 \%$ of buildings and structures in the residential area of the town are photovisualized (Fig. 2, 3) [10]. 


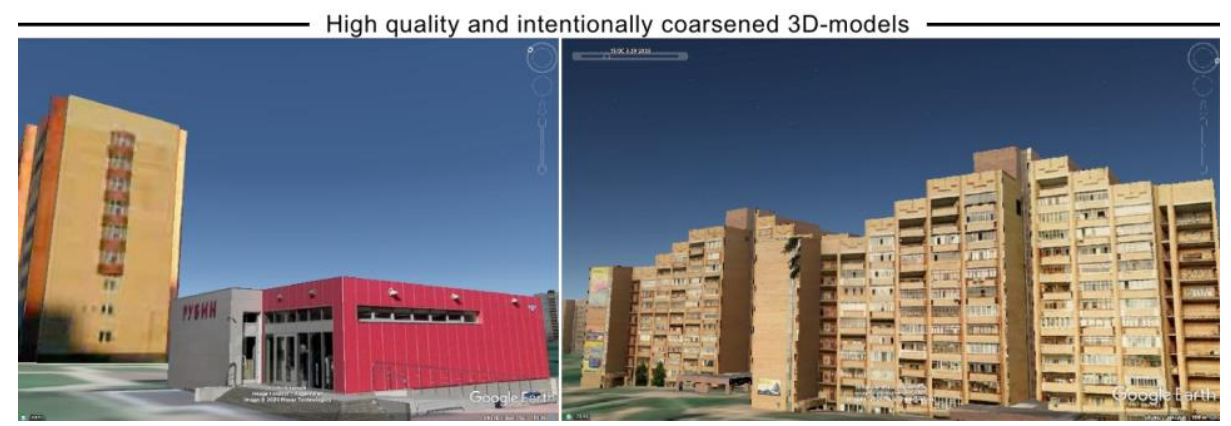

Fig. 3. (a, b). (Left to right) Photo-visualization of the 3D-models. a) Normal quality (foreground), coarsened quality (background), and b) intentionally coarsened buildings.

\subsection{Spatially specified medical and ecological data}

The aim of the ongoing research is a comprehensive study of the social situation in the town and, above all, its medical and environmental aspect with the highest possible spatial and temporal resolution through the widespread use of scientific visualization [11].

The ultimate goal of the present stage is to collect empirical data and achieve spatialtemporal resolution of the "apartment- a-day". At present, the resolution of a "residential house-a-day" is achieved.

The data set on infectious diseases in Protvino for the five-year period (2011 - 2016) - a total of 3791 records with registered location (Fig. 4) - is used in the study. Different approaches were used for visualizing the data: cartographic visualization by the means of vector layers, density maps ("heat maps"), direct 3D- and 4D-visualization, etc. In this study density maps (Fig. 6) were compiled in QGIS software.

For preliminary assessment of the environmental factor the data of social and hygienic monitoring were used - content of carbon monoxide, sulfur dioxide, aerosol particles, nitrogen dioxide in the atmosphere, gamma radiation levels observed in 2010 (Fig. 5). The data were presented online on the site of the Protvino 3D model and supplemented with a layer of data on unauthorized garbage dumps identified in the surrounding Protvino forests. There are no significant variations of the parameters in the residential area of the Protvino due to its small area and lack of local sources of the pollution. 
8 E. Eremchenko, V. Tikunov, S. Vylegzhanin, et al.

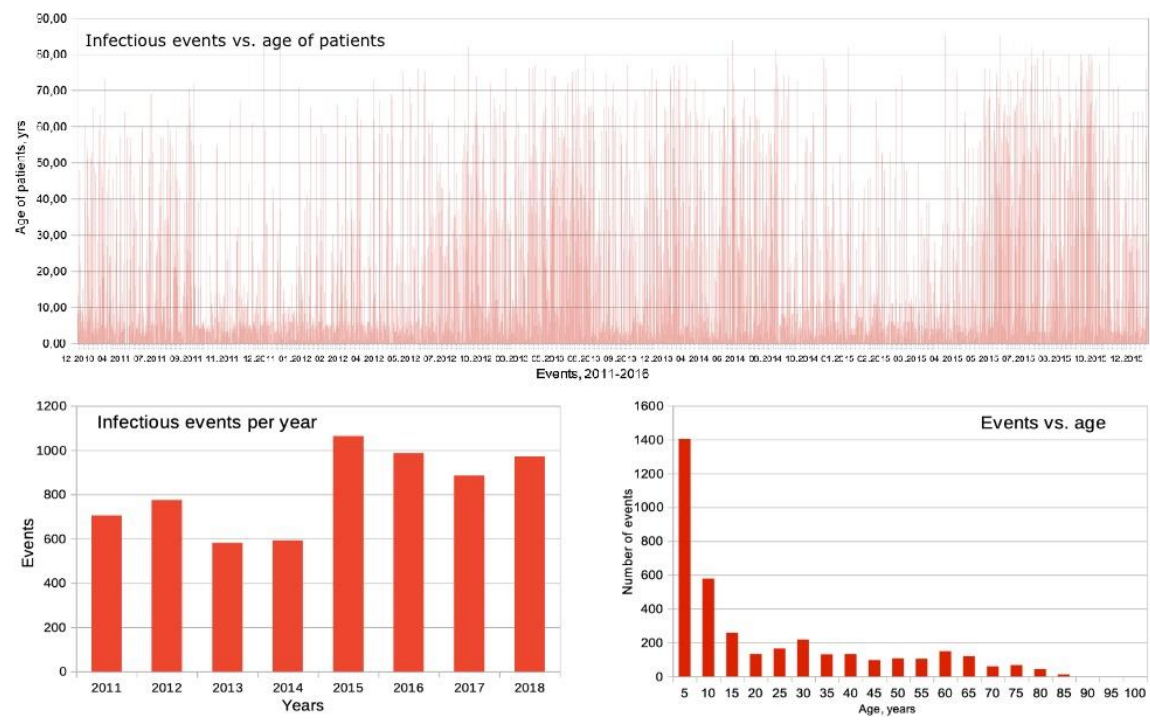

Fig. 4. (a - c). (Up to down, left to right). a) Age of infectious patients, 2011-2016. b) Infectious events per year. c) Number of events vs. age of patients.

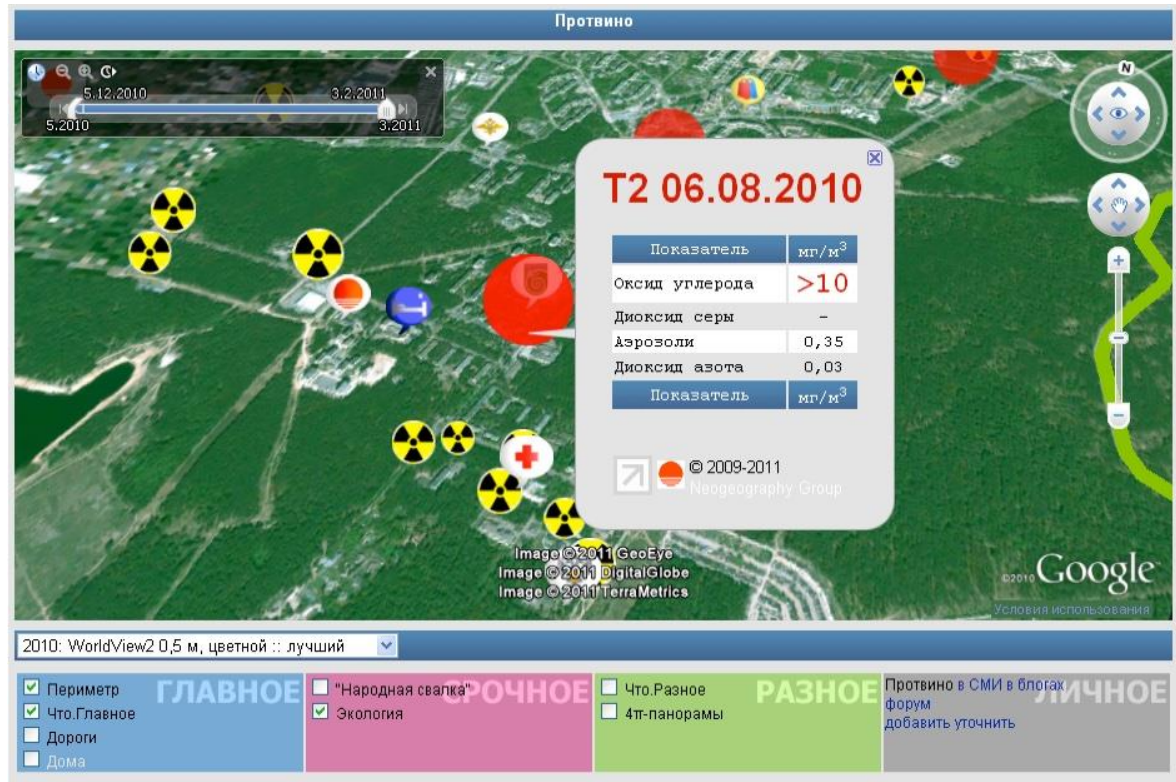

Fig. 5. Web visualization of ecological data in the 3D-model of Protvino. 
The visual analysis of the data with the help of scalar density representation (Fig. 6, ad) unveils that some of the residential buildings in the Protvino residential zone characterized by abnormally high levels of infectious diseases, which clearly distinguish them from neighboring buildings. Also there is a residential area consists of three separate buildings of about 2.5 thousand residents (near $7 \%$ of the city population) with unexpectedly low levels of infectious diseases (Fig. 7 a, b). Variations in infection rates in Protvino are characterized by the high level of variability in indicators even for neighboring buildings, which makes it difficult to explain the spatial dynamics observed as a result of the impact of generalized environmental factors - like pollution of air, for example.

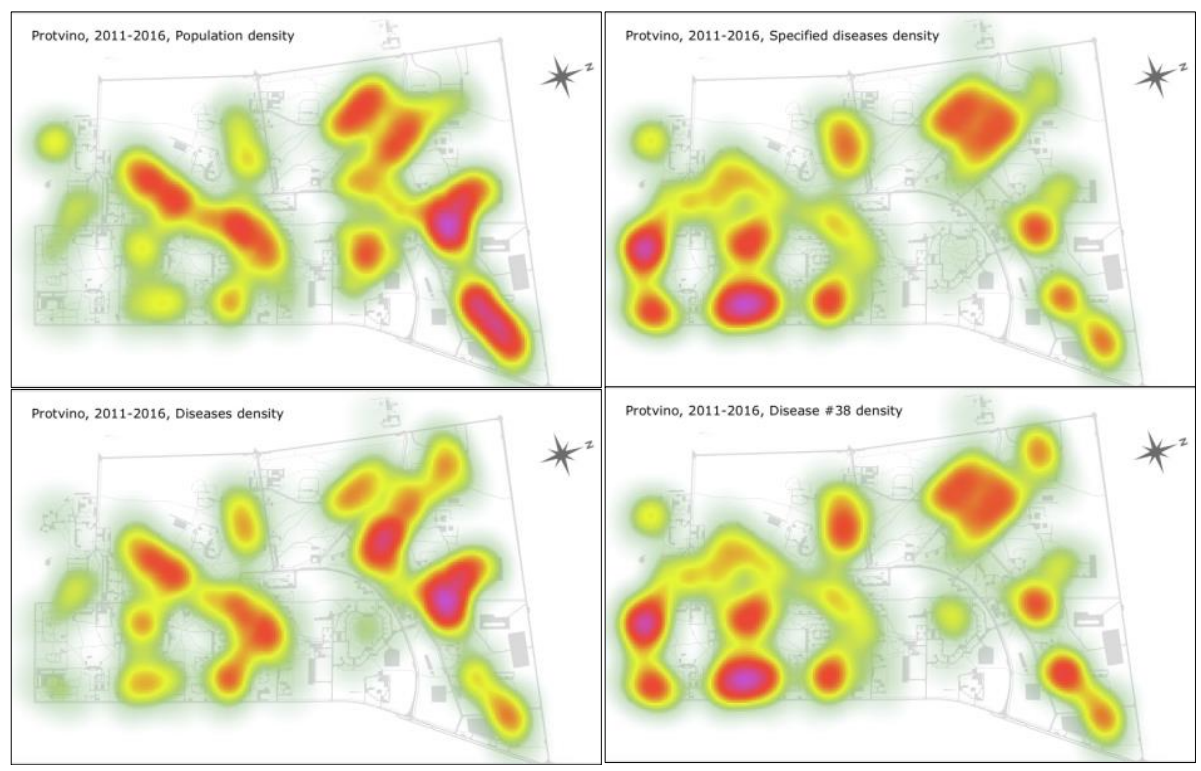

Fig. 6. (a - d). (Left to right, up to down). a) Population density of Protvino. b) Specified diseases density in Protvino. c) Diseases density in Protvino. d) Disease \#38 density in Protvino.

Therefore, the high heuristic potential of spatial data and the possibility of using them to develop a model for describing the process and forming a context is also demonstrated.

\subsection{Spatially non-specified medical and ecological data}

The difficulty in explaining the observed intense dynamics of infectious diseases in a small modern town with variations of specific environmental factors makes it natural to set the opposite task - to analyze non-specific global and regional factors for a given 
10 E. Eremchenko, V. Tikunov, S. Vylegzhanin, et al.

area. An excellent basis for such an analysis were provided by the COVID-19 pandemic, which allowed collecting a significant relevant data set on the development of a global infectious process with high spatial and temporal resolution $[12,13]$.

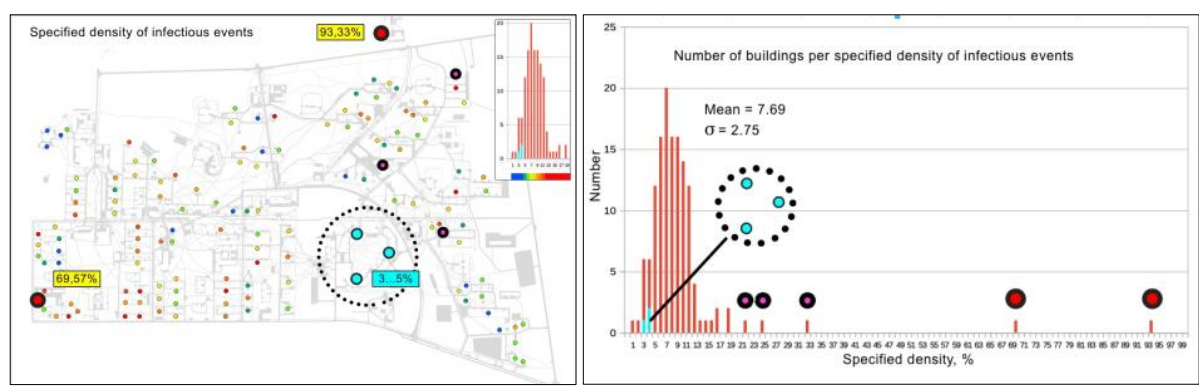

Fig. 7. (a, b). (Left to right). Specified density of infectious diseases, most healthy and most affected residential buildings, represented a) in the space, and b) on the histogram.

Analysis shows that the global temporal dynamics of the pandemic over a long period (17 weeks) were characterized by monotony, stability and an overall exponential increase in the number of new cases, overlaid with clearly distinguishable weekly variations (Fig. 7a). At the same time, within the same period in the U.S., which was the main contributor to the cases and gave up to half of the total daily statistics, there was at least a twofold change of trends to the opposite ones (steady decline - rapid growth steady decline), due to the internal specifics of the process development and changing of local rules and countermeasures (Fig. 7b). The same striking difference bet ween rapidly changed local trends and sustainable global trend is observed in other countries that make a major contribution to the statistics of COVID-19 pandemic.

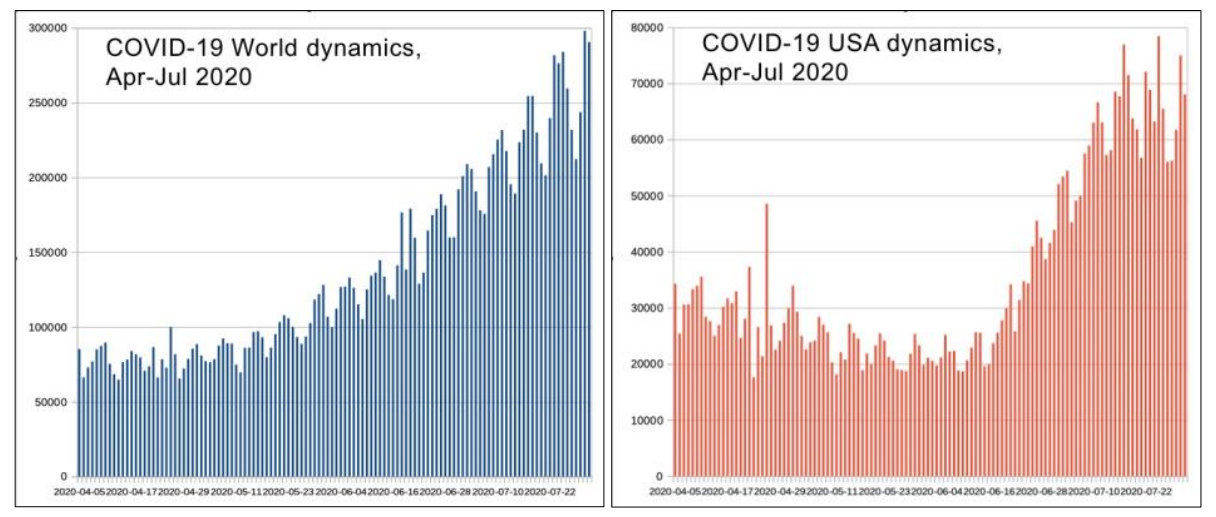

Fig. 4. (a, b). (Left to right). a) COVID-19 World dynamics, April-July 2020. b) COVID-19 USA dynamics, April-July 2020.

Surprisingly, such significant changes did not affect the overall global dynamics of the epidemic, as they were compensated by simultaneous, but oppositely oriented changes 
in the dynamics of the process in all other territories. This allows us to conclude that the dynamics of a given pandemic do not consist of isolated process development results in different countries under the influence of local factors, but are regulated by the global factor and if the process dynamics in a certain territory is affected, it is compensated by the opposite influence in other territories of the globe.

This conclusion means that the development of the epidemic process in the local area will be determined not only by a set of local factors - both medical and ecological. It also regulated by the global factor that support the sustained global trend. This, in turn, means that the study of correlations between the development of epidemic processes in different regions of the Globe and at different scales, which re-quires the creation of models in the paradigm of the Digital Earth, is highly desirable.

\section{Conclusions}

Due to a combination of factors, the interpretation of the results of monitoring of the infection dynamics and ecological factors in the urban environment requires critical considerations. The main problem is relevance and integrity of source data, and lack of the data at all. The absence of theoretical models for the development of social and, in particular, epidemic processes complicates their interpretation, and the intermediate spatial and temporal resolution achieved so far further limits the heuristic potential of already available materials. In particular, it will be necessary to analyze the development of infections in multistory buildings and study the temporal aspect of the dynamics of infectious diseases.

Due to the need to take into account both specific and non-specific factors in the development of infectious processes, seamless integration of data for different areas becomes a mandatory methodological condition of research. Support for inter-scale topology becomes necessary. This requires the use of the Digital Earth as a platform for research on infectious processes.

The problem of data privacy will be a significant problem in achieving "apartment/day" resolution, as direct visualization allows the clearly recognizable association of any event with a specific apartment and/or house in the visual context. Ensuring the effective use and high precision visualization of information with the protection of personal data and while maintaining its heuristic potential will be a separate challenge for the next phase of the research [14].

On the other hand, even the available data with the use of effective methods and approaches can significantly expand our understanding of the development of epidemic processes in urban environments in general and in a particular locality. We can already state that such dynamics is characterized by significant spatial gradients and can hardly be explained by the hypothesis of the impact of any environmental factors deliberately generalized in space - for example, the content of impurities and gases in the air or the quality of water pipes. On the contrary, the analysis of non-specific features of global epidemics allows us to speak about the existence of a global factor that determines the development of the epidemic in individual territories. 
12 E. Eremchenko, V. Tikunov, S. Vylegzhanin, et al.

\section{References}

1. Ud Din, R., Shah, K., Ahmad, I. et al.: Study of transmission dynamics of novel COVID-19 by using mathematical model. Adv Differ Equ 2020, 323 (2020).

1. Castronovo, D., Chui, K., \& Naumova, E.: Dynamic maps: a visual-analytic methodology for exploring spatio-temporal disease patterns. Environ Health 8, 61 (2009).

2. Stout Cameron M.: GIS as a Tool in the Study of Diseases: The Great Cholera Outbreak in the Soho District of London, England (1854). ESRI Canada User Conference, p. 24. Ottawa (2011).

3. Baturin Yu., Eremchenko E., Zakharova M.: 3D-document and Digital Earth. CEUR Workshop Proceedings 29th International Conference on Computer Graphics and Vision GraphiCon, Vol. 2485, pp. 155-158, BTU, Bryansk (2019).

4. Google Earth, http://earth.google.com, last accessed 2020/06/12.

5. Gore A.: The Digital Earth: Understanding our planet in the 21st Century, http://www.digitalearth-isde.org/userfiles/The_Digital_Earth_Understanding_our_planet_in_the_21st_Century.doc, last accessed 2020/07/25.

6. Li, Z., Yan, H.: Transformation in Scale for Continuous Zooming. In: Guo, H., Goodchild, M., Annoni, A. (eds.) Manual of Digital Earth. Springer, Singapore (2020).

7. Szabo ,S., Tache, Y., Somogyi, A.: The legacy of Hans Selye and the origins of stress research: A retrospective 75 years after his landmark brief "Letter" to the Editor\#ofNature. Stress, 15(5), pp. 472-478. (2012).

8. Protvino 3D-model, http://vprotvino.ru, last accessed 2020/06/12.

9. Wolodtschenko, A., Eremchenko, E., Klimenko, S. et al.: 2005-2015. Istoriya razvitiya 3Dmodeli Protvino (Russian). Naukograd: Nauka, Proizvodstvo, Obshchestvo 1(3), pp. 20-25. (2015).

10. Aleshin, A., Afanasiev, A., Brusentsev, P., et al.: Modern information technology: Information visualization, virtual environment, neo-geography, tangible images. Scientific Visualization, volume 5, issue 4, pp. 1-17. (2013).

11. COVID-19 dashboard by the Center for System Science and Engineering (CSSE) at John Hopkins University (JHY), https://gisanddata.maps.arcgis.com/apps/opsdashboard/index.html\#/bda7594740fd40299423467b48e9ecf6, last accessed 2020/08/03.

12. Owid/covid-19-data. https://github.com/owid/covid-19-data/tree/master/public/data/, last accessed 2020/08/03.

13. Eremchenko, E., Fetishev, A., Vylegzhanin, S.: High-precision monitoring of the epidemic situation with the help of 3D-model of the settlement. Geocontext. v. 6, pp. 48-57 (2018). 